\title{
FROM NICHE TO MAINSTREAM TRANSITIONS: DIGITAL SECOND-HAND MARKETS FOR MILLENNIALS
}

\author{
Swati Srivastava and Alma Leora Culén \\ Department of Informatics, University of Oslo \\ PB 1080, 0316 Blindern, Oslo, Norway
}

\begin{abstract}
This paper addresses the issue of over-consumption among millennials by repositioning reacquisition of consumer goods and digital platforms that support peer-to-peer exchange. We use transition design to frame our thinking and provide the focus on local communities and global technologies. To understand millennials, we have used in-depth interviews as the main method. The analysis of the interview data led to the recognition of five significant barriers for the uptake of second-hand platforms. Synthesis of findings, together with consumer behavior archetypes that we have identified previously, led to nine implication for the design of future services. These are a significant step toward re-conceptualization of peer-to-peer services and building of local communities of interest based on lifestyles and interests, including being greener.
\end{abstract}

\section{KEYWORDS}

Sustainable Services, Transition Design, Socio-Cultural Factors, Second-Hand Markets, Green Choices

\section{INTRODUCTION}

In this paper, we describe a real-life collaborative project with industrial partners interested in more sustainable consumption and the new business models that support it. We approached the project with the transition design framework (Escobar, 2018; Irwin, 2015, 2018; Manzini, 2015) and aimed to understand how to mitigate over-consumption amongst millennials.

Transition design is an emerging field of design, based on deeper entanglement of design with socio-cultural factors and systemic thinking, technology and future-oriented positioning. In proposing transition design, Irwin states that "transition to sustainable futures will require a sweeping change at every level of our society" (Irwin, 2015). Furthermore, the success of transformational change "will depend upon our ability to change our ideas about change itself - how it manifests and how it can be initiated and directed" (ibid.). Transition design, thus, advocates the re-conception of entire lifestyles, based on a deep understanding of underlying systems dynamics, aiming to make them place and community-based. It forwards four main areas in which design and research activities unfold to scaffold transitions in positive directions: visions of the future, finding and using the appropriate theories of change, understanding internal mindset and values, as well as external attitudes, and finally, discovering and using new ways of designing.

While the thinking behind our methodological approach is novel, our method involving in-depth interviews is well proven. With fifteen millennials we discussed their present consumption habits and the use of digital re-distribution (second-hand markets) platforms. The analysis of interviews played a crucial role in the proposal of design strategies based on thinking ecologically and including social, cultural and sustainability perspectives. In line with transition design, these strategies are to actively engage young people in re-thinking over-consumption and 'throw away' culture that they are a part of. Millennials represent the segment of the population inheriting serious global problems that span every aspect of human life, from environmental to social. They are already living in times of change, and are increasingly engaged in creating alternatives to present day consumer practices, e.g., (Peyer, Balderjahn, Seegebarth, \& Klemm, 2017), but these are still far from mainstream ideas. We have specifically focussed on millennials, who often have a strong role as influencers for other age groups (Prensky, 2001; Selwyn, 2013). 
The research presented here was done at the beginning of our collaboration with the industrial partners. However, contrary to the habitual linear paths of dissemination of academic results, it was not published first. The design practice outcomes were shared with the community first, where the main outcome was a future oriented service. The assemblage of design outcomes, i.e., the set of artefacts representing the service concept and supporting the service, was exhibited at a conference (Srivastava, 2017). An academic paper discussing the service (Srivastava \& Culén, 2018) was also published. Furthermore, we have discussed the value of finding consumer behaviour archetypes and using this knowledge to support transitions to more sustainable consumption in (Srivastava \& Culén, 2019).

The findings from the interviews that are presented in this paper, provided the basis to discuss opportunities regarding the re-positioning of the digital second-hand markets from niche to mainstream, and from global to place and community-based ones.

In what follows, we first outline the background work that helps us to position and frame this research towards transitioning to decreased consumption of consumer goods among millenials. We then discuss the findings from the interviews, focusing exclusively on challenges and opportunities to reduce consumption using digital platforms and novel services. We outline implications for design of future services, within the context of digital re-acquisition services featuring everyday things, and scaffolding local connections and community building.

\section{BACKGROUND}

Starting from transitional thinking (aiming to make a shift from todays to better consumption practices on digital platforms), we present the transition design framework first, then look briefly at sustainable consumption research within interaction design. We also provide a short summary of our research on finding consumers behavioural archetypes based on their current consumption practices and lifestyles. Together, these provide the conceptual background for the work and the way to contextualize our findings.

\subsection{Transition design}

Irwin defines transition design as "a proposition for a new area of design practice, study, and research that advocates design-led societal transition toward more sustainable futures”, (Irwin, 2015). To frame this practice, four areas of design and research activities are foundational for scaffolding transitions in a positive direction. Those are visions of the future, finding and using the appropriate theories of change, understanding internal mindset and values, as well as external attitudes, and finally, discovering and using new ways of designing (Escobar, 2018; Irwin, 2018; Lockton \& Candy, 2018; Tonkinwise, 2015), see Figure 1.

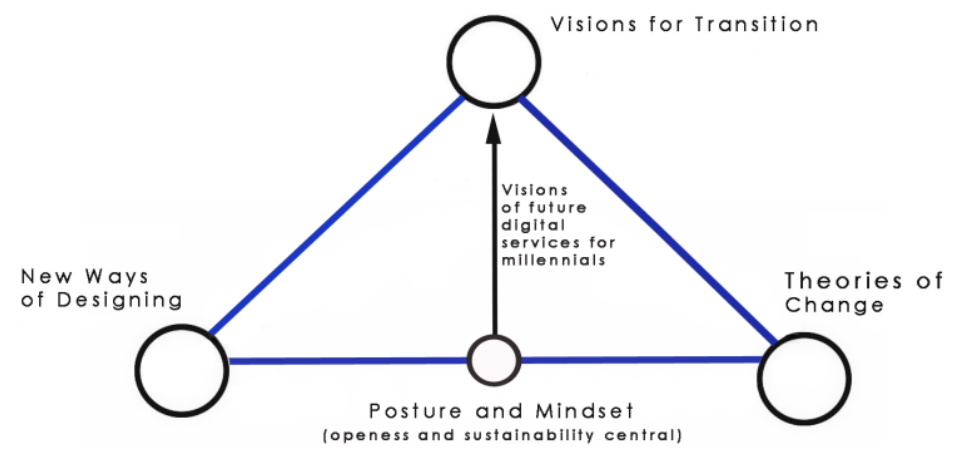

Figure 1. Transition Design Framework to transform consumer practices. Source: modified from (Irwin, 2018)

Furthermore, as any other design activity, transition design oscillates between the concrete, every day actions, and the more abstract theories and concepts and explorations, thus actively combining the research and the design. This is reminiscent of the processes described by Fallman (Fallman, 2008) for research through design, but what sets transition design apart is the intent to effectuate large scale societal changes. 
The necessary changes from the perspective of transitions thinking involve changes in lifestyles and socio-cultural norms, and as such are more complex and challenging. The role of transition designers is to act as transformation agents, enabling thinking and action towards change. However, sustainability transitions need to be addressed by cross-disciplinary approaches in which design and designers stands together with different scientific disciplines needed to address the challenges at hand, as well as other types of knowledge that can lead towards finding better ways to frame the solution space in terms of plural opportunities (Escobar, 2018; Leach et al., 2012).

\subsection{Sustainable Consumption Practices in Interaction Design}

Social practice theories are particularly suitable for supporting sustainability transitions, focusing on horizontal trajectories and interconnectedness of everyday, place based practices (Ingram, Shove, \& Watson, 2007; Shove, Pantzar, \& Watson, 2012). To understand and engage people in re-thinking situated practices, (understood as routinized and everyday activities in some context), it is helpful to frame them as a unit of design. This way of looking at practices, since introduction in (Kuijer, Jong, \& Eijk, 2013), has been gaining traction.

Using social practice theories as a way to understand how to change people's behaviors, has made its way in sustainable interaction design work. For example, Pierce and Paulos discuss consumption and frame it as practices related to acquisition, possession, dispossession and reacquisition, exploring reacquisition (purchasing used, second-hand goods) in particular, as a possible pathway toward more sustainable consumption practices (Pierce \& Paulos, 2011). They point to an important distinction between designing for or with communities of reacquisition, and designing from communities of reacquisition. The former has to do with improving reacquisition as practices (better technologies, services, more profound concerns for the environment), while the latter engages in processes and practices of reacquisition as the basis for seeking and shaping of new solutions. The latter relates well to social practice theories, and thus was well in tune with our previous choices of designing for transitions using social practice as a theory of change. Their rich qualitative study of reacquisition informed on the importance of values, social acceptance, and challenges with current practices of reacquisition. Furthermore, it brought attention to the relation between dispossession and reacquisition, and the importance of experientially desirable acquisition. Also, in (Odom, Pierce, Stolterman, $\&$ Blevis, 2009), authors discuss dispossession and reacquisition, focusing on what things mean and outlining four areas of importance for relations between things and people: engagement (with things), histories (personal stories, memories), augmentation (creative ways of using, enhancing) and perceived durability.

\subsection{Behavioral Archetypes}

Behavioral archetypes are understood as behaviors carried forward from deep historical roots that are used to anchor socio-cultural norms. We subscribe to a culturally coded view of the archetype, i.e., archetypes are understood as iconic representations of social meanings and behavioral norms. In (Srivastava \& Culén, 2019), we have identified three basic archetypes that millennials' consumption patterns follow. All three highlight the transient character of their living situation as the most important factor that shapes their purchase decisions, such as buying brand new or second-hand goods. We named these three categories Spirited Nurtured Dwellers (age 16 - 19, lived at home with their parents), Busy Frugal Nomads (age $20-23$, moved out of their parents' home into the temporary living situations such as collectives or the student housing), and Steady Independent Movers (age 24 - 27, employed and building their careers). We point out to their mutual inter-connectedness and interdependences, and underlying transience as the key motivation behind the engagement with re-distribution services within this demographic. Thus, we identified the opportunity to explore ways of scaffolding transience by visioning different transitions and relations between the archetypes, creating pathways for movements of well-functioning consumer goods, but also knowledge, skills and time, within a familiar, similar-minded demographic. This can provide a foundation for new local, community-based social practices, leading to prolonged life of goods and a more sustainable consumption. 


\section{METHOD}

We approached the issue of consumption among youth with a mindset of openness: we wished to do a broad, open, reflective and exploratory inquiry into routinised behaviours, focusing archetypal understanding of youth and their consumption practices around second-hand goods, and particularly, second-hand digital markets and platforms.

The method of choice were semi-structured in-depth interviews. We engaged fifteen young people ( 7 males and 8 females), see Table 1, in conversations lasting between one and two hours. The interviews were recorded, anonymized, then transcribed and coded using emergent, thematic coding. The analysis started with each author coding transcripts of two interviews and then discussing the emerging themes to help guide the remaining work with content analysis.

Table 1. The table provides the participants' pseudonyms, gender and age. The archetypes are color-coded

\begin{tabular}{|c|c|l|c|c|c|l|l|}
\hline$\#$ & G & Pseudonym & Age & 8 & F & Nomadine-3 & 21 \\
\hline 1 & F & Dwelline-1 & 17 & 9 & M & Nomad-4 & 19 \\
\hline 2 & F & Dwelline-2 & 16 & 10 & M & Mover- 1 & 27 \\
\hline 3 & M & Dweller-3 & 17 & 11 & F & Moverine-2 & 26 \\
\hline 4 & F & Dwelline-4 & 16 & 12 & F & Moverine-3 & 24 \\
\hline 5 & F & Dwelline-5 & 16 & 13 & M & Mover-4 & 24 \\
\hline 6 & M & Nomad-1 & 22 & 14 & M & Mover-5 & 24 \\
\hline 7 & F & Nomadine-2 & 21 & 15 & M & Mover-6 & 26 \\
\hline
\end{tabular}

The analysis presented in this paper focuses on the use of digital platforms and design implications for future digital services.

\section{FINDINGS}

Several categories of findings emerged through he analysis. The largest category constitutes challenges related to trust, positioning of the market as the place to get rid of things, on-line presentation of goods, difficulties with communication, and the effort that it takes to find things.

\subsection{Perceptions of the Existing Services and Platforms}

The majority of the existing digital reacquisition services are peer-to-peer systems. Consequently, the participants' experiences with such services and platforms focused primarily on peer-to-peer interactions, necessity of engaging in direct communication and exchange of assets, representing contextually situated social challenges. In addition, second-hand markets are culturally coded as places where one gets rid of things, not as a re-distribution of quality things. These traits of the peer-to-peer economy for re-distribution of second-hand goods pose a very real set of challenges for millennials.

The social challenges had to do with issues of trust and familiarity. They preferred to avoid transactions when profiles seemed very different from their own age group, citing unfamiliarity, security and a sense of general awkwardness as primary reasons. For instance, Dwelline-2 considers someone who is at the university, and a student, to be trustworthy and close to her own profile: "I am going to the university next year. I would gladly buy a used book if there are not too many scribblings and notes in it. I am open to that because the person I would buy it from would be a trustworthy student from the same campus."

When asked why she believes that a person she would meet for such exchange is more trustworthy, she replied: "I guess the age, it matters a lot." The issue of trust is sensitive and Dwelline-2 thinks that people close to her own age are in principle more trustworthy because "I do not trust old men. I do not know why. Something stereotypical, probably. Maybe, when I see pictures of their faces, I am judgmental, I don't know, but maybe I am."

While Dwelline-2 did not have a direct experience with peer-to-peer transactions, but was open to it under certain conditions, Nomadine-3 has the first-hand experience. However, certain sensibility around trust was 
present in her experience as well: "Yeah, I bought a lamp from a guy who lived on my block. I went to his place, because he was not living far away. I could just walk there. I didn't think that this was a [safety] problem for me, because he was also talking to another guy who wanted to buy. When the other guy didn't come through, he decided to sell to me". Elaborating on safety issues, Nomadine-3 added:

"When I'm on FIND [the largest online platform called Finn ('finn' means find, see finn.no) in Norway], I check the seller. But not like, 'Okay, he looks like a particular kind of a person.' Some pictures do make me more sceptical regarding the seller, but it is not very often I feel that. But for me, he [the lamp owner] just seemed like an okay person. He was nice, and also, when I was going to his place, I think he was returning home from work or something. So, I just came up to his flat and waited at the door until he arrived."

Participants expressed some hesitance when considering exchange within own social circles. For most of the participants this hesitance stemmed from the risk of making their friends uncomfortable by, for example, unintentional spamming with advertisements on social media, which could affect their relations and reputation within the social circle.

"If I was selling something expensive, I would like to tell my friends and family on Facebook, and they could get a lower price. I wouldn't post my clothes or things like that on Facebook, because I think that would be like spamming them. It's personal. Small stuff. If I was selling a boat or something, I would let people know. Or a car", Nomad-1.

When asked further, if he thought that people do not care in general about the stuff he is selling, or they do not care for him doing it on the Facebook, Nomad-1 explained: "Both actually. If you want something second-hand, you go on FIND. You don't want to get your social media spammed with advertisements. It already has enough ads."

These few examples illustrate inclinations to trust people of their own age more, but not within own social circles, where they are afraid of being judged negatively. The middle ground was found to be young people who were not friends, but lived in their neighbourhood, or in a place they know. They were situated between the extreme familiarity (friends and family) and non-familiarity. Open profiles on FIND allowed for trust judgments, but this openness added a time and effort consuming task of filtering profiles, potentially leading to an overall less positive experience.

Furthermore, a prevailing perception of second-hand platforms was that of a market for unwanted things. This perception is reinforced by services providers themselves by advertising their services as means of getting rid of things, and making easy money and space for first-hand purchases. This led to a creation of a vicious circle regarding positioning of the market as a place to get rid of things. This perception was very evident among our young participants. For instance, Mover-1 describes the sale of very expensive shoes very cheaply on FIND by saying: "I just wanted to get rid of them." Many other participants used the exact same wording, and admitted to being motivated by the intent to 'get rid of things', which Moverine-3 describes as "It can be a bit difficult because part of why people put up things on FIND is just so that someone else will get rid of them [instead of disposing of things themselves]."

While most of the participants expressed willingness to buy, use and sell functional goods at reduced prices at online platforms, these remained largely unpopular within this age group. When the re-distribution platforms were used, and thought of in more positive ways, it was related to making fast money, personal gain, niche hobbies or treasure-hunts. Not one participant thought of these digital platforms as platforms representing greener choices.

\subsection{Stories, Attachments, Quality of Goods}

The participants admitted to being willing to accept minor visible flaws as a trade-off for acquiring things at a lower price, as long as the flaws were clearly communicated by the seller. However, along with the current state of goods, and clarity in conveying it, the participants underlined that if the flaw has an interesting story behind it, the story was important to their decision-making process. They recounted several instances where they themselves worked to discover the story behind the artefact of interest. Examples provided told stories about purchases from a famous person, a brand item at an unbelievable price, or a brand-new artefact at a low price. Even if the presentation of the artefact on the platform had an interesting story, distinct content or style, it was found to increase their interest. Furthermore, if the attachment of the previous owner to the item was visible, it also has a positive effect. As Mover-6 pointed out "You need to know that it [a musical 
instrument in this case] has been taken care of, its been dealt with properly and not misused because its about to become something personal for me, yeah, so that is an aspect I look at."

Many other participants also explained that they limited their purchases to objects they had expertise on, or experience with, as it allowed them to judge its condition easily. Most reacquisition services, naturally, do not offer any guarantee on the object itself. Therefore, for many users, an open platform with little familiarity with the community influences their trust not only in people, but also in the durability/quality of the goods.

\subsection{Communication and (In)Conveniences}

It was clear from the interviews that none of the participants were looking to build social relationships through second-hand platforms, it was not seen as a means to meet new people. They expected (and sometimes wanted) a cordial transaction and were open to the idea of a serendipitous social connections but it was not a reward they anticipated from the engagement. However, most of the participants cited communication as one of the key inconveniences of the process.

"You have to meet up with this person. You have to decide, 'Oh, we can meet on this day, then you can give me the money, I'll give you the clothing.' Just setting that up that meeting, it is... I don't know. I don't want to do it".

When selling, they preferred minimal communications. Rather than answering requests and inquiries from multiple users, they preferred to be able to connect with one or two potential buyers and concluding the transaction fast and with minimum negotiations.

\subsection{Lack of Reliability, Commitment, and Information}

A common concern raised by most of the participants was a lack of support of any kind on second-hand platforms, besides affordance to connect. Even though expectations, in contrast to the first-hand platforms, were lower, figuring to out every step of the way on their own can quickly became time-consuming. For instance, the decision-making process to sell or buy second-hand requires information gathering on its demand and estimated selling price in the market. This further requires spending time identifying and checking out similar listings. The participants have to decide on a selling price, evaluate the user profiles, the logistics of carrying things, mutually deciding a meetup times, and negotiating modes of monetary transactions. While the second-hand marketplace is often presented as an instant and easily accessible platform to buy or list/sell disused items (mainly because of their presence as smartphone apps), engaging in a transaction in this marketplace carries along many uncertainties. The drop-offs (a point of failure) in the all services available at present, also played a significant role in deterring the participants from investing time in reacquisition. Participants having experience with reacquisition platforms said that making a commitment to make a transaction and setting time aside for it did not mean that the other party would show up as agreed, and sometimes things were sold, but the change in status not updated on the app (a common concern). Moverine- 3 expresses these common concerns as follows:

"Sometimes I've tried to buy things, but they have been sold. I just wish I could have had an overview of the transaction. It would have been better because sometimes you ask the person: 'Hey can I buy that thing?' They are like 'Yeah, it's still available.' Then you say 'Cool, when can I pick it up?' You then never hear from that person again. Then you're like, 'Hey, I haven't heard from you.' And then they say 'Oh, I sold it to someone else'. I just feel 'What? Why? We had a deal!'”

Moverine-3 expresses frustration with such communication and the lack of transparency. However, she is almost conflicted when she justifies such a bad user experience as an acceptable trade-off for getting things at a considerably lower price than at the first-hand markets. It points towards an implicit positioning of sellers over buyers, adding to the lack of accountability and transparency in the transaction. Mover-1 points to the following:

"In a way, I was expecting some help, with putting it [the item] in the car and everything. No such help, it was like okay, put the money there and get rid of that, you're helping me get rid of that. Practically, she paid me for the job of getting rid of it, I wasn't paying her for the table. I was actually paying her for doing a small job for her, but then that's why the price was really, really cheap. It was easier for her to get rid of the table in this way." 
This implied that sellers view transactions as a free labour with a small profit, not as a value of the item. Unlike large peer-to-peer systems such as Uber, or Airbnb, most re-distribution platforms do not rate sellers, freeing them from the pressure of earning ratings or reputation, resulting in a lack of ongoing commitment.

\subsection{Invisibility of the Market}

Engagement with digital reacquisition marketplaces remains a deliberate act within this age group. Unlike looking at storefronts that can give a sense for the goods available in the first-hand market, discovering the second-hand market remains effort-demanding and requiring an intentional and planned activity involving high screen-time. Mover-1 explains that he is not willing to do this work. Instead, he says: "That would be my girlfriend [who is willing to put in the effort]. I prefer to buy new things just because I don't deal with this. Also, even if you went to find used stuff, you still need to go to particular places. Together, that's more time consuming for me."

This is quite ironic since desired second-hand goods and the marketplace are often present in the user's physical locality, close to home. Still, even a casual exploration of the second-hand inventory demands careful and undivided user attention. The invisibility of the market, and effort of discovery required, often presented a barrier to engagement with second-hand markets, even when the possibility of saving money, getting access to durable goods and making greener choices are all considered.

\section{IMPLICATIONS FOR DESIGN AND DISCUSSION}

We now draw on the finding to outline the implications for design, where our vision focussed on designing for wider uptake of reacquisition, within the mainstream communities of teens and young adults.

\section{Re-positioning reacquisition within the existing practices and transient lifestyles of millennials}

As evident from the interviews, the practices of possession and dispossession of everyday consumer goods pivot around transient living situations. Reacquisition services have the potential to offer larger assistance to sustainably manage the flow of consumer goods, instead of using a generic peer-to peer approach of simply connecting the buyers and the sellers. Rather than designing a prescriptive solution for a seamless point-to-point peer transaction, redistribution services should offer a holistic system addressing the material needs and challenges of the dynamic, aspirational, and busy lifestyles of this young demographic. Going beyond the possibility of making greener choices, monetary benefits, and riddance, a reacquisition service should be built to connect peers with familiar needs rituals/practices around usage of possessions.

\section{Design to create pathways for movements of things to a context of value}

Pierce et. al. emphasise the context as a means of re-coding the value of domestic objects and the potential of digital technologies to facilitate the articulating this intent (Pierce \& Paulos, 2011). The pivotal position of both the context as well as the digital medium is evident in our research as well. Building on this, we explore the agency of design in building a robust pathway for the movement of durable goods across meaningful contexts. We argue for designing the path of reacquisition by connecting peers with complimentary lifestyles as well as a familiar routine of use.

\section{Simplifying research and evaluation through intelligent information curation}

Discovery and time-effort needed to identify desirable items are important barriers for many young users of reacquisition services. However, intelligent data processing systems are becoming increasingly common, opening new opportunities also in relation to reselling platforms. Sophisticated algorithms can reduce the manual evaluation process at the user's end, by capturing details and recognizing patterns and predicting outcomes. This can lead to a highly customized and relevant marketplace for all, reducing the time investment.

\section{Design to eliminate the reputation of "easy riddance"}

Lack of quality control in second-hand platforms' inventory was one of the common concerns amongst all the participants. It has a cascading negative effect on the service experience. Given the subjective nature of acceptance criteria of goods amongst the users, a clinical quality check alone cannot manage this challenge. 
Reselling systems need to be designed and branded to eliminate their reputation of being platforms for "getting rid of unwanted things". The subtle inclination of these services towards selling as opposed to buying, through narratives and systems interactions encourages the use of these platforms as a convenient disposal strategy as well as a source of cheap throwaway goods, countering the intent of reducing consumption. Instead, reselling platforms need to be designed to adopt and promote the narrative of movement/circulation rather than riddance of unwanted things, where the community views the goods as a representation of their intents, practices, and aspiration. Additionally, by positioning the service as a clear and defined platform for mainstream utilitarian goods for the younger population with a transient lifestyle, the quality standards can be tailored towards maintenance and monitoring the peers and the service providers.

\section{Design to engage durable brands directly with reacquisition}

Durable inventory is fundamental to reacquisition services aiming to promote sustainable consumption. However, preventing the inflow of nondurable goods, in these services can be immensely challenging. A possible step in resolving this issue is for reacquisition services would be to be use branding and design to make goods having a longer lifespan an essential part of their identity, moving consciously away from the popular strategy of riddance of unwanted things. Durable brands can potentially utilize second-hand marketplace to advertise the high build quality of their offerings. With intelligent algorithms becoming more and more commonplace, this change in the brand identity and strategy can be supported through effective quality control and management of the inventory.

\section{Design for positive social friction amongst the users}

As reflected in the interview accounts, on the one hand, social interactions required for reselling transactions were deemed uncomfortable and unwanted by an otherwise usually social user group of millennial and digital natives. At the same time, the participants also admitted to these interpersonal conversations being essential to both product and peer evaluation. A sustained and positive social friction can assist in building trust and familiarity in a peer-to-peer service, especially in the case of reacquisition services where the engagement of both the buyers and sellers are often infrequent and need-based. Designed for engagements in addition to merely buying and selling can encourage a sense of community and possibly leading to other peripheral social interactions between the users. For instance, reacquisition market platforms can also function as forum for the young millennials to assist each other settling down in a new city, or offering reviews on second hand values and lifespan of various goods.

\section{Design for a visible market}

Making the availability of second-hand goods in the locality more prominent is important. The issue of time is essential, and a more mainstream positioning of reacquisition services requires a more visible presence of the goods available locally, to reduce the efforts of searching through a large inventory. For instance, customized ads of curated goods for individuals in their university campus can make the available inventory more visible through the use of technology beyond mobile and web platforms. To be sure, we do not necessarily imply that second-hand markets need to emulate the first-hand model of opening physical shops and showrooms. However emerging technology like connected objects and augmented reality can be used to make the inventory 'visible' in inventive ways. This can enable the youth easily and contextually discover second-hand objects and the marketplace in general while going about their lives.

\section{Design for closed platforms and curated community}

Closed platforms imply the control over who is a member of the peer-to-peer service. It allows for the community to be shaped through connections and familiarity and enables organic building of trust and empathy leading to more reliability and commitment to the community. It implies setting new norms, recoding the culture of the platform, defining clearly what participants should expect from the other members, people worth similar and complimentary intents and lifestyle participate and interact with other with the intent of helping out each other and also fulfilling their needs of domestic goods.

\section{Design to support the environmental/green motivations}

The interviews show that millennials are motivated to make greener choices, and second-hand market places can use that as a leverage point. The services should clearly and visibly communicate this intent and focus on this issue in a transparent and factual way. The design itself needs to convey the honest efforts to support sustainable practices. 


\section{CONCLUSION}

Conducting in-depth interviews with 15 young people, we have discovered five significant barriers for the uptake of second-hand services among millennials. These have served as starting points for synthesis that led to the articulation of nine implications for future services. To implement the findings in the real world, cross-disciplinary collaboration is needed to discuss and implement them, as well as chart the best ways to achieve transition visions toward more sustainable consumption by millennials. The results of this paper are a significant step in understanding how to re-conceptualize services and allow them to support communities of interest in ways that are in line with their lifestyles and interests, including the motivation to be greener.

\section{ACKNOWLEDGEMENT}

This work was conducted as part of the research and innovation project Conserve and Consume, funded by the Norwegian Research Council (project number 235526/O30).

\section{REFERENCES}

Escobar, A. (2018). Designs for the Pluriverse: Radical Interdependence, Autonomy, and the Making of Worlds. Durham: Duke University Press Books.

Fallman, D. (2008). The Interaction Design Research Triangle of Design Practice, Design Studies, and Design Exploration. Design Issues, 24(3), 4-18.

Ingram, J., Shove, E., \& Watson, M. (2007). Products and Practices: Selected Concepts from Science and Technology Studies and from Social Theories of Consumption and Practice1. Design Issues, 23(2), 3-16.

Irwin, T. (2015). Transition Design: A Proposal for a New Area of Design Practice, Study, and Research. Design and Culture, 7(2), 229-246.

Irwin, T. (2018). The Emerging Transition Design Approach. DRS 2018: Catalyst, Volume 3, pp. 968-989.

Kuijer, L., Jong, A. de, \& Eijk, D. van. (2013). Practices as a Unit of Design: An Exploration of Theoretical Guidelines in a Study on Bathing. ACM Trans. Comput.-Hum. Interact., 20(4), 21:1-21:22.

Leach, M., Rockström, J., Raskin, P., Scoones, I., Stirling, A. C., Smith, A., Thompson, J., Millstone, E., Ely, A., Arond, E., Folke, C., \& Olsson, P. (2012). Transforming innovation for sustainability. Ecology and Society, 17(2):11.

Lockton, D., \& Candy, S. (2018). Vocabulary for Visions in Designing for Transitions. DRS 2018: Catalyst, Volume 3, pp. $908-926$.

Manzini, E. (2015). Design, When Everybody Designs: An Introduction to Design for Social Innovation (R. Coad, Trans.). Cambridge, Massachusetts: The MIT Press.

McDonald, S., \& Oates, C. J. (2006). Sustainability: Consumer Perceptions and Marketing Strategies. Business Strategy and the Environment, 15(3), 157-170.

Odom, W., Pierce, J., Stolterman, E., \& Blevis, E. (2009). Understanding why we preserve some things and discard others in the context of interaction design. Proceedings of the SIGCHI Conference on Human Factors in Computing Systems, 1053-1062.

Peyer, M., Balderjahn, I., Seegebarth, B., \& Klemm, A. (2017). The role of sustainability in profiling voluntary simplifiers. Journal of Business Research, 70, 37-43.

Pierce, J., \& Paulos, E. (2011). Second-hand Interactions: Investigating Reacquisition and Dispossession Practices Around Domestic Objects. Proceeding of SIGCHI Conference on Human Factors in Computing Systems, 2385-2394.

Prensky, M. (2001). Digital Natives, Digital Immigrants Part 1. On the Horizon, 9(5), 1-6.

Selwyn, N. (2013). The digital native - myth and reality. Aslib Proceedings. https://doi.org/10.1108/00012530910973776

Shove, E., Pantzar, M., \& Watson, M. (2012). The dynamics of social practice: Everyday life and how it changes. Sage.

Srivastava, S., 2017. Zygo: Design led reframing of secondhand marketplaces, Nordes: Design + Power. Exhibit.

Srivastava, S., Culén, A.L., 2018. Transition-oriented Futuring: Integrated Design for Decreased Consumption amongst Millennials, DRS 2018: Catalyst, Volume 3, pp. 927-940.

Srivastava, S., Culén, A.L., 2019. Implications for Transitions to Sustainable Consumption: Finding Millennials' Behavior Archetypes. Forthcoming.

Tonkinwise, C. (2015). Design for Transitions - from and to what? Design Philosophy Papers, 13(1), 85-92. 